\title{
Absorbing state phase transition with competing quantum and classical fluctuations
}

\author{
Matteo Marcuzzi, ${ }^{1}$ Michael Buchhold, ${ }^{2}$ Sebastian Diehl, ${ }^{2}$ and Igor Lesanovsky ${ }^{1}$ \\ ${ }^{1}$ School of Physics and Astronomy, University of Nottingham, Nottingham, NG7 2RD, United Kingdom \\ ${ }^{2}$ Institut für Theoretische Physik, Universität zu Köln, D-50937 Cologne, Germany
}

(Dated: May 25, 2016)

\begin{abstract}
Stochastic processes with absorbing states feature examples of non-equilibrium universal phenomena. While the classical regime has been thoroughly investigated in the past, relatively little is known about the behavior of these non-equilibrium systems in the presence of quantum fluctuations. Here we theoretically address such a scenario in an open quantum spin model which in its classical limit undergoes a directed percolation phase transition. By mapping the problem to a non-equilibrium field theory, we show that the introduction of quantum fluctuations stemming from coherent, rather than statistical, spin-flips alters the nature of the transition such that it becomes first-order. In the intermediate regime, where classical and quantum dynamics compete on equal terms, we highlight the presence of a bicritical point with universal features different from the directed percolation class in low dimension. We finally propose how this physics could be explored within gases of interacting atoms excited to Rydberg states.
\end{abstract}

PACS numbers: 64.70.qj, 32.80.Ee, 31.15.xk

Introduction. - Non-equilibrium phenomena can be found in many different contexts, ranging from chemical reactions to disease-spreading. Analogously to the equilibrium case, non-equilibrium ensembles can show the emergence of universal behavior, signaling the irrelevance of the microscopic details of the dynamics for macroscopic observables. This occurs when such outof-equilibrium systems start to act collectively [1-4]. A distinction can be made depending on the presence or absence of detailed balance [5] 8 , between systems which evolve towards a stationary equilibrium state 9] (e.g., quenched systems coupled to thermal baths [10]) or that preserve their non-equilibrium character even in the longtime limit, representing flux equilibrium states.

Directed percolation (DP) 11 constitutes an instance of a classical phase transition to an absorbing state, i.e., a state which can be reached, but not left by the dynamics, and represents a simple instance of a broader class of intrinsically non-equilibrium phase transitions [11 14]. Despite its robustness, its experimental observation has so far been elusive [15, with a single exception [16, 17. However, it was recently suggested to realize and explore DP dynamics in cold gases of atoms excited to high-lying Rydberg states [18. In this work, we harness the opportunities that result from the fact that Rydberg gases are actually open quantum systems to go beyond the realm of classical physics (see also [19]), and establish a generalised absorbing state phase transition in the presence of quantum fluctuations. Driven-dissipative systems constitute indeed an ideal platform for the investigation of the interplay between classical and quantum effects, and have been recently addressed in a broad range of experiments. The spectrum includes light-driven semiconductor heterostructures [20, arrays of driven microcavities [21, 22], cold atoms in optical lattices [23], cavities [24, 25] and microtraps $[26,28]$. Several among these instances employ excitation of the atoms to high-lying Rydberg orbitals [29 31] in order to achieve strong interatomic interactions and to study cooperative effects 32 36.

In these systems, the driving/dissipation not only introduces coherence loss, but also explicitly violates the equilibrium conditions at the microscopic level [7, 37. It is thus a challenge to identify to what extent the nonequilibrium and quantum nature of the dynamics impact on the macroscopic phase diagram and phase transition properties. Oftentimes, upon coarse graining such systems lose their quantum character and equilibrium conditions are effectively restored [38 43. But there are instances where non-equilibrium [44, 45] and quantum [46, 47] aspects persist even at asymptotically large wavelength. The transition we highlight here does not fall into the DP universality class, and its origin can be unambiguously traced back to the presence of coherent dynamics. More precisely, the latter introduces a first-order non-equilibrium phase transition without counterpart in the purely classical DP problem. This discontinuous phase transition terminates in a bicritical point which even asymptotically at large distances and in dimensions $d<2$, does not feature the symmetries underlying DP, or any equilibrium problem.

Model. - We reproduce a quantum variant of the contact process (for an introduction see [11). Basically, it consists of a lattice of "active" and "inactive" sites, where the former can spontaneously decay to inactive, whereas activation can only occur in the proximity of already active sites. Thus, the fully-inactive state is absorbing. Specifically, we consider a lattice of quantum two-level systems with spacing $r$. On every site $k$ we define the basis $\left|a_{k}\right\rangle$ (active) and $\left|i_{k}\right\rangle$ (inactive), the density of active sites $n_{k}=\left|a_{k}\right\rangle\left\langle a_{k}\right|$ and the ladder operators $\sigma_{k}^{+}=\left|a_{k}\right\rangle\left\langle i_{k}\right|$ and $\sigma_{k}^{-}=\left|i_{k}\right\rangle\left\langle a_{k}\right|$. Under the action of Markovian noise sources, the state $\rho$ of the sys- 




Figure 1. (Color online) (a) Fundamental processes. We consider a lattice whose sites admit two states: active (red) and inactive (green). Active sites decay to inactive at a rate $\gamma$. Proliferation of active sites is possible through classical (rate $\kappa)$ and quantum (strength $\Omega$ ) branching. (b) One-dimensional $(z=2)$ phase diagram constructed from the effective action (5) in saddle-point approximation (color code corresponds to density of active sites). All parameters are measured in units of $\gamma$. In the classical limit $(\Omega=0)$ the system exhibits a continuous (2nd order) directed percolation phase transition between an absorbing state and a finite-density one. This transition extends into the quantum regime (thick red line) up to the critical point $\alpha$. In the quantum limit $(\kappa=0)$ a first-order transition is found which also extends into the classical regime (dashed yellow line) up to point $\alpha$. In the neighborhood of this line, a narrow region of coexistence of two attractive stationary solutions is present, which is not resolved here. The high values of the density reached in the active phase stem from neglecting higher orders in $n$ in the action, which would otherwise enforce $n \leq 1 / 2$.

tem evolves according to the Lindblad equation [48, 49] $\dot{\rho}=-i[H, \rho]+\sum_{a, k} \mathbf{D}\left[L_{a, k}\right] \rho$ [see sketch in Fig. 1], where

$$
H=\Omega \sum_{k} C_{k} \sigma_{k}^{x} \quad \text { with } \quad C_{k}=\sum_{j \mathrm{nn} k} n_{j}
$$

is the quantum Hamiltonian, $\sigma_{k}^{x}=\sigma_{k}^{+}+\sigma_{k}^{-}$, and "nn $k$ " denotes nearest neighbors (nn) of site $k$; $\mathbf{D}[X] \rho=X \rho X^{\dagger}-\left(X^{\dagger} X \rho+\rho X^{\dagger} X\right) / 2$ is the dissipator and $L_{a, k}$ are the so-called jump operators, with indices $a$ (process type), and $k$ (lattice site). These jump operators are chosen to define a modified contact process [11, which is known to feature a DP transition, and include decay $L_{\mathrm{d}, k}=\sqrt{\gamma} \sigma_{k}^{-}\left(\left|a_{k}\right\rangle \rightarrow\left|i_{k}\right\rangle\right)$ and - for every neighbor $j$ of $k$ - branching $L_{\mathrm{b}, j, k}=\sqrt{\kappa} n_{j} \sigma_{k}^{+}$(an active site can activate a neighboring one $\left.\left|a_{j} i_{k}\right\rangle \rightarrow\left|a_{j} a_{k}\right\rangle\right)$ and coagulation $L_{\mathrm{c}, j, k}=\sqrt{\kappa} n_{j} \sigma_{k}^{-}$(the inverse process $\left.\left|a_{j} a_{k}\right\rangle \rightarrow\left|a_{j} i_{k}\right\rangle\right)$. The "constraint" operator $C_{k}$ in $H$ represents the simplest choice reproducing the requirement of an active site nearby to flip a spin; this makes $H$ the "minimal quantum equivalent" of the noisy branching/coagulation above. Similar constrained Hamiltonians have been studied in the past with a focus on quantum glassy behavior [19] and many-body localization [50, 51].

Equations of motion and density path integral. - We infer here the properties of the phase diagram by exploiting an effective path integral description for the density variable $n_{k}$ alone. We start by deriving the HeisenbergLangevin equations of motion (EOM) 52] for the singlesite operators $n_{k}, \sigma_{k}^{x}$ and $\sigma_{k}^{y}=-i \sigma_{k}^{+}+i \sigma_{k}^{-}$. For convenience we introduce the coordination number $z$ (number of nearest neighbors per lattice site) and the shorthand $P_{k}^{x / y}=\sigma_{k}^{x / y} \sum_{j \mathrm{nn} k} \sigma_{j}^{x}$. In the following, we also measure all times and energies in units of $\gamma$, i.e., we set $\gamma=1$ :

$$
\begin{aligned}
\dot{n}_{k} & =-n_{k}+\left[\Omega \sigma_{k}^{y}-\kappa\left(2 n_{k}-1\right)\right] C_{k}+\hat{\xi}_{k}^{n}, \\
\dot{\sigma}_{k}^{x} & =\Omega P_{k}^{y}-\frac{z \kappa+1}{2} \sigma_{k}^{x}-\kappa \sigma_{k}^{x} C_{k}+\hat{\xi}_{k}^{x}, \\
\dot{\sigma}_{k}^{y} & =\Omega P_{k}^{x}-\frac{z \kappa+1}{2} \sigma_{k}^{y}-\left[\Omega\left(4 n_{k}-2\right)+\kappa \sigma_{k}^{y}\right] C_{k}+\hat{\xi}_{k}^{y} .
\end{aligned}
$$

The quantum noise terms $\hat{\xi}_{k}^{\alpha}$ consider the fluctuations of the bath and depend on the structure of the jump operators. They show vanishing averages but non-trivial, Markovian correlations, which for the present setup are (in rescaled units) $\left\langle\hat{\xi}_{k}^{x} \hat{\xi}_{k^{\prime}}^{x}\right\rangle=\left\langle\hat{\xi}_{k}^{y} \hat{\xi}_{k^{\prime}}^{y}\right\rangle=\delta_{k, k^{\prime}},\left\langle\hat{\xi}_{k}^{n} \hat{\xi}_{k^{\prime}}^{n}\right\rangle=$ $\delta_{k, k^{\prime}} n_{k},\left\langle\hat{\xi}_{k}^{x} \hat{\xi}_{k^{\prime}}^{y}\right\rangle=-i \delta_{k, k^{\prime}},\left\langle\hat{\xi}_{k}^{n} \hat{\xi}_{k^{\prime}}^{x}\right\rangle=-\delta_{k, k^{\prime}} \sigma_{k}^{+}$and $\left\langle\hat{\xi}_{k}^{n} \hat{\xi}_{k^{\prime}}^{y}\right\rangle=i \delta_{k, k^{\prime}} \sigma_{k}^{+}$up to leading order in the density [53].

In the following, we work in the continuum limit $(k, t) \rightarrow(\vec{x}, t) \equiv X$ and derive an effective path integral for the density field $n_{X}$ via a Martin-Siggia-Rose (MSR) construction [3, 56 58, presented in the supplemental material [53. Crucially, the $\sigma^{x, y}$-fields are gapped, and thus can be integrated out perturbatively. The resulting long-wavelength field theory depends on the density variable $n$ alone, and is obtained by additionally performing a derivative expansion of the action. It reads

$$
\begin{aligned}
S_{n} & =\int_{X} \tilde{n}_{X}\left[\left(\partial_{t}-D \nabla^{2}+\Delta\right) n_{X}+u_{3} n_{X}^{2}+u_{4} n_{X}^{3}\right] \\
& -\int_{X}\left[\frac{1}{2} \tilde{n}_{X}^{2} n_{X}+\mu_{4} \tilde{n}_{X}^{2} n_{X}^{2}\right] \equiv S_{n}^{(1)}+S_{n}^{(2)},
\end{aligned}
$$

where $D=r^{2} \kappa$ represents a diffusion constant and $\Delta=1-z \kappa-\frac{8 z^{2} \Omega^{2}}{(z \kappa+1)^{3}}, u_{3}=2 z\left(\kappa-\frac{2 z \Omega^{2}}{z \kappa+1}\right), u_{4}=\frac{8 z^{2} \Omega^{2}}{z \kappa+1}$ and $\mu_{4}=\frac{2 z^{2} \Omega^{2}}{(z \kappa+1)^{2}}+\frac{128 z^{4} \Omega^{4}}{(z \kappa+1)^{6}}$ are the microscopic coupling constants. The response field $\tilde{n}$ encodes the linear response properties of $n$ under small perturbations.

We emphasize two key properties of the action (5): First, the absence of a density-independent Markovian noise level $\sim T \tilde{n}_{X}^{2}$ (necessarily present in classical systems in thermal equilibrium). This is characteristic of DP dynamics, which feature the absence of density fluctuations in the absorbing state $n_{X}=0$ and consequently 

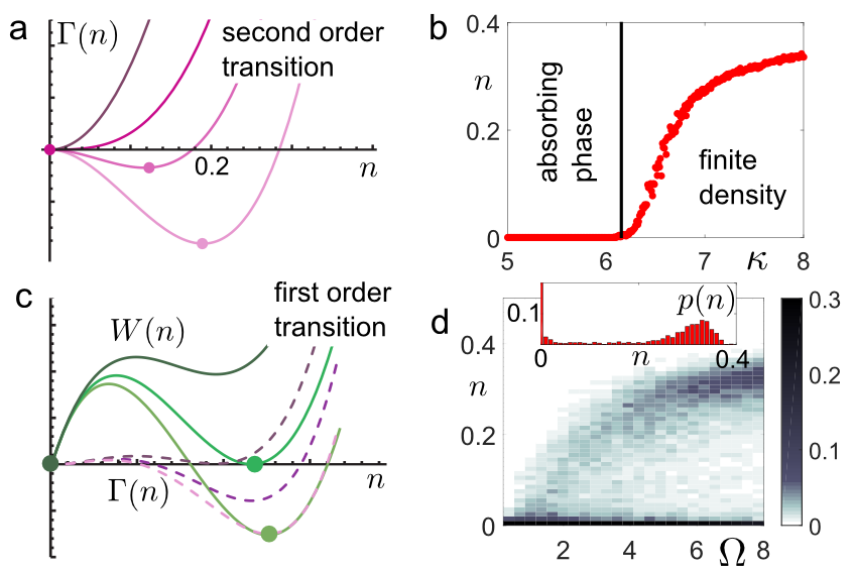

Figure 2. Effective potential and phase transitions. (a) Behavior of the effective potential $\Gamma(n)$ (arbitrary units) across the second order phase transition. Dots mark the minima of $\Gamma(n)$. The transition occurs when $\Delta$ in Eq. (6) changes sign. (b) Stationary state density in the classical limit $(\Omega=0)$ as a function of $\kappa$ (chain of 200 sites, average over $10^{3}$ realizations per point), obtained via Monte Carlo simulations starting from a completely active configuration and stopped at time $\gamma t=10^{4}$. The data show the characteristic behavior of a continuous phase transition around $\kappa_{c} \approx 6.2$. (c) Effective potential $\Gamma(n)$ (dashed lines) and corresponding "optimal-path" potential $W(n)$ (solid lines), see Eq. 77, across the first-order transition. At the transition point, $W\left(n_{1}=0\right)=W\left(n_{2}\right)=0$. (d) Steady-state histogram of the density in the quantum limit $\kappa=0$ (12 spins) obtained via a quantum-jump Monte Carlo (QJMC) method, indicating a first-order transition $\left(\Omega_{c} \approx 2\right)$ as $\Omega$ increases. Two stable stationary solutions, one with zero and one with finite density, emerge. The inset displays a section of the histogram taken at $\Omega=8$.

a multiplicative kernel $\propto n_{X}$. An additive noise introduced by the dissipative terms $L_{d}=\sqrt{\gamma} \sigma^{-}$only occurs in the eliminated spin variables $\sigma^{x, y}$. Second, the presence of a non-zero coherent coupling $\Omega \neq 0$ - i.e. the intrinsic quantum effect - leads to the appearance of nonzero couplings $u_{4}$ and $\mu_{4}$ as well as a negative contribution to $u_{3}$. This additional "quantum" scale $\Omega$ breaks a fundamental symmetry of the DP class (specified below) and strongly modifies the phase diagram compared to the purely-dissipative model [see Fig. 1].

Effective potential and mean-field phase diagram. The discussion of the various phases and transitions of the system is considerably simplified by realizing that the deterministic contribution to the action $S_{n}^{(1)}$ can be written as $\int_{X} \tilde{n}_{X}\left[\partial_{t} n_{X}-D \nabla^{2} n_{X}+\frac{\delta \Gamma\left(n_{X}\right)}{\delta n_{X}}\right]$, where

$$
\Gamma(n)=\frac{\Delta}{2} n^{2}+\frac{u_{3}}{3} n^{3}+\frac{u_{4}}{4} n^{4}
$$

is a local effective potential. In the absence of fluctuations $\Gamma$ characterizes the mean-field phases, which are determined by the properties around its minima.

The corresponding phase diagram is shown in Fig. 1(b). The active phase is identified by $\Delta<0, u_{4} \geq 0$ and $u_{3}>0$, which leads to a single minimum of the effective potential at finite density. On the other hand, when both $\Delta$ and $u_{4}$ are positive, there is a local minimum of $\Gamma$ at $n=0$. For negative and sufficiently strong cubic coupling $u_{3}<-2 \sqrt{u_{4} \Delta}$, there exists a second local minimum at finite density $n>0$. In this regime, the mean field evolution features two attractive fixed points and the thermodynamic phase is determined within the optimal path approximation in phase space [59].

Three different types of phase transitions from the active to the inactive state can be thus identified, their nature depending on the specific choice of parameters and the dimensionality. When the gap $\Delta$ vanishes with both $u_{3}, u_{4}>0$ the system undergoes a second order phase transition [see Fig. 2(a)], corresponding to a diverging correlation length $\xi=1 / \sqrt{|\Delta|} \rightarrow \infty$. Numerical evidence for this transition is presented in panel (b) of Fig. 2, which displays the stationary density of active sites obtained for $\Omega=0$ in a chain of 200 sites. For $\Delta>0$ and $u_{3} \leq-2 \sqrt{u_{4} \Delta}$, the transition from the active to the inactive phase takes place instead at finite correlation length $\xi=1 / \sqrt{|\Delta|}<\infty$. The form of the effective potential $\Gamma(n)$ suggests a first-order transition line in this regime featuring the coexistence of the zero and finite-density solutions. This case, however, requires additional care due to the specific form of the noise, as detailed further below.

The $\alpha$ point in Fig. 11(b) located at $\Delta=u_{3}=0$ represents a bicritical point at which both the line $(\Delta>$ $\left.0, u_{3}=-2 \sqrt{\Delta u_{4}}\right)$ and the line of continuous transitions $\left(\Delta=0, u_{3}>0\right)$ terminate. At this point, the quartic potential term $u_{4}$ provides the leading non-linearity.

Fluctuations at the continuous transition. - The competition between quantum and classical dynamics strongly affects the nature of the active-to-inactive transition. In the absence of the coherent coupling, $u_{4}, \mu_{4}=$ 0 , the action (5) is equivalent to the so-called Reggeon field theory for classical DP [60. It features - upon rescaling the fields - the characteristic rapidity inversion symmetry, which leaves the system invariant under the transformation $n \leftrightarrow-\tilde{n}$ and $t \rightarrow-t$ [3, 7, [59]. For $u_{4}>0$, this symmetry is broken by the microscopic action. The implications depend on the dimension $d$ : For $d>2, u_{4}$ is RG irrelevant and can be discarded in the infrared-dominated dynamics close to the continuous transition. Consequently, in $d>2$, rapidity-inversion is restored and the line of continuous transitions displays universal scaling behavior corresponding to classical DP.

At the $\alpha$ point [white dot in Fig. 1 (b)], $u_{3}=0$ and the leading-order coupling becomes $u_{4}$. For $d>2$, the continuous transition at this point is governed by mean-field scaling behavior, since $u_{4}$ is RG-irrelevant and cannot introduce infrared divergent corrections to the vanishing couplings $u_{3}, \Delta$. On the other hand, for $d<2, u_{4}$ becomes RG relevant and generates a non-trivial RG flow of $\Delta$ and $u_{3}$ on the entire second order transition line. 
This leads to a violation of rapidity-inversion which persists at long wavelength, and thus drives the system away from the DP critical point to a different non-equilibrium universality class, without specific symmetries. In $d<2$, therefore, only the isolated point $\kappa=1 / z, \Omega=0$ lies in the DP class, while the presence of quantum fluctuations imprints a new universal scaling behavior on the entire line, including the $\alpha$ point. In $d=2$, the scaling of the fluctuation corrections to $u_{4}$ determines whether this coupling becomes relevant, making the scenario equivalent to $d<2$, or irrelevant, which has to be determined by an RG analysis.

Non-equilibrium discontinuous transition. - For $(\Delta>$ $\left.0, u_{3}<-2 \sqrt{\Delta u_{4}}\right)$ the effective potential $\Gamma$ displays two distinct minima, $n_{1}=0$ and $n_{2}=\frac{\left|u_{3}\right|}{2 u_{4}}+\left(\frac{u_{3}^{2}}{4 u_{4}^{2}}-\frac{\Delta}{u_{4}}\right)^{1 / 2}$, suggesting a first-order phase transition. The actual transition line lies where the finite-density minimum becomes statistically preferred. In equilibrium, this would be the point at which the minima of $\Gamma$ are at the same height. However, the present non-equilibrium noise shows more pronounced fluctuations at larger densities and thus favors $n_{1}$ over $n_{2}$. To estimate the steady state distribution function $P(n)$, we apply the optimal path approximation to the action 3 , 59; this involves treating the coefficient $\Xi(n)=\frac{1}{2} n+\mu_{4} n^{2}$ of $\tilde{n}^{2}$ as a kind of mean-field, density-dependent temperature. It yields 53.

$$
P(n)=\frac{1}{Z} e^{-V W(n)}, \text { with } W(n)=\int_{0}^{n} d m \frac{\partial \Gamma / \partial m}{\Xi(m)},
$$

with volume $V$ and normalization $Z$. Both potentials $W(n)$ and $\Gamma(n)$ vanish in $n_{1}$ and share the finite-density minimum $n_{2}$. In the thermodynamic limit $V \rightarrow \infty$, $P(n) \rightarrow \delta\left(n-n_{l}\right)$, where $l=1,2$ depending on which one is the global minimum of $W$, accounting for the physical constraint $n \geq 0$. The transition occurs when $W\left(n_{2}\right)=0$, which identifies the non-equilibrium firstorder line [dashed line in Fig. 1 (b)]. Due to the nonequilibrium nature of the fluctuations, this does not coincide with the naive prediction $\Gamma\left(n_{2}\right)=0$, as shown in Fig. 2(c). In Fig. 2(d) we report the full-counting statistics of the density $n$ obtained via QJMC techniques 61] for a chain of 12 spins. Despite the presence of strong finite-size effects, a bimodal structure is still highlighted for large values of $\Omega$. This implies that trajectories bunch together around two possible values, the absorbing one and a finite-density one, and is a signature of the aforementioned coexistence.

Realization with Rydberg atoms. - Atoms excited to Rydberg states are employed in current experiments to study many-body effects [26, 32, 34, 62,70. Recently, several theoretical studies addressed the semiclassical limit of these systems [71, 72] connecting their dynamics to that of constrained classical ones [72, 73]. Reasoning along the same lines of Ref. 18, we discuss below an implementation which should permit the exploration of the physics discussed above.
The internal structure of Rydberg atoms can be approximated as a ground state $\mid$ GS $\rangle \equiv|i\rangle$ (inactive site) and an excited one $|\mathrm{Ryd}\rangle \equiv|a\rangle$ (active site). Rydberg gases feature strong van-der-Waals interactions in state |a) 29 31, which rapidly decay as $r^{-6}$ with the interparticle distance $r$. For the sake of simplicity, we approximate them here as nearest-neighbor terms $V_{\mathrm{nn}}$ in a one-dimensional configuration.

Quantum branching/coagulation is realized via coherent driving by a laser field of Rabi frequency $\Omega$ and detuning $\Delta_{L}$ with respect to the atomic transition frequency; fixing $\Delta_{L}=-V_{\mathrm{nn}}$ enables an "anti-blockade" $71,74,75$, mechanism which favors the excitation of a Rydberg atom next to an already excited one, e.g. $|i a i\rangle \rightarrow|i a a\rangle$. Differently from the idealized model above, the constraint requires here a single excitation nearby, and processes such as $|a i a\rangle \rightarrow|a a a\rangle$ are highly suppressed. The Hamiltonian is therefore approximately given by $H_{\mathrm{ryd}}=$ $\Omega \sum_{k} C_{k}^{\prime} \sigma_{k}^{x}$ where $C_{k}^{\prime}=n_{k-1}+n_{k+1}-2 n_{k-1} n_{k+1}$.

To generate the incoherent branching/coagulation the atoms are coupled (with coupling $g$ ) to a second equallydetuned light field with strong phase noise (dephasing rate $\lambda \gg g$ ) [76]; for a correlation length shorter than the interatomic distance, the bath is modeled as independent bosonic modes $b_{k}, b_{k}^{\dagger}$ acting on each lattice site. The effective equation of motion for the atoms is obtained by performing second order perturbation theory in the small parameter $g / \lambda[18,77$, 78]. The resulting master equation for the reduced atomic density matrix $\rho$ is

$$
\dot{\rho}=\frac{4 g^{2}}{\lambda} \sum_{k}\left(\left\langle b_{k}^{\dagger} b_{k}\right\rangle \mathbf{D}\left[C_{k}^{\prime} \sigma_{k}^{+}\right]+\left\langle b_{k}^{\dagger} b_{k}+1\right\rangle \mathbf{D}\left[C_{k}^{\prime} \sigma_{k}^{-}\right]\right) \rho .
$$

For sufficiently high $\left(\left\langle b_{k}^{\dagger} b_{k}\right\rangle \gg 1\right)$ and homogeneous $\left(\left\langle b_{k}^{\dagger} b_{k}\right\rangle \approx\left\langle b_{m}^{\dagger} b_{m}\right\rangle\right)$ intensity, one can identify $\kappa=$ $\left(4 g^{2}\left\langle b_{k}^{\dagger} b_{k}\right\rangle\right) / \lambda$, leading to the branching/coagulation jump operators: $L_{\mathrm{b}, k}^{\mathrm{ryd}}=\sqrt{\kappa} C_{k}^{\prime} \sigma_{k}^{+}$and $L_{\mathrm{c}, k}^{\mathrm{ryd}}=\sqrt{\kappa} C_{k}^{\prime} \sigma_{k}^{-}$. The final process is radiative decay from the Rydberg state to the ground state, with jump operator $L_{\mathrm{d}, k}^{\text {ryd }}=$ $\sqrt{\gamma} \sigma_{k}^{-}$[31].

Although the microscopic formulation of the dynamics is slightly different from the previously-discussed model in particular, atoms with more than one excited neighbor are brought off-resonance - the resulting phase structure is similar, as the EOMs only differ from Eqs. (2) by RG-irrelevant higher-order density terms.

Outlook. - We have investigated the effects of quantum dynamical processes on a prototypical absorbingstate phase transition. We highlighted the emergence of a richer structure in the phase diagram, which includes both a discontinuous and a continuous non-equilibrium transition. In low dimension $d<2$ the presence of a quantum coherent process leads to a breaking of the only fundamental symmetry of DP in a way that persists at long wavelengths, and thus leads to a phase transition 
of a different nature. In equilibrium, the interplay between classical (thermal) and quantum fluctuations typically leads to a dimensional crossover [2, 79]. The present work shows that out of equilibrium the picture is not as straightforward and opens the path for further investigations in this field, including the quantitative characterization of the new universality class.

Acknowledgments. - M.M. and I.L. wish to express their gratitude for the insightful discussions with J.P. Garrahan and for access to the University of Nottingham High Performance Computing Facility. I.L. acknowledges that the research leading to these results has received funding from the European Research Council under the European Union's Seventh Framework Programme (FP/2007-2013) / ERC Grant Agreement n. 335266 (ESCQUMA). Further funding was received through the H2020-FETPROACT-2014 grant No. 640378 (RYSQ) and from EPSRC Grant no. EP/M014266/1. M. B. and S. D. acknowledge funding by the German Research Foundation (DFG) through the Institutional Strategy of the University of Cologne within the German Excellence Initiative (ZUK 81).

[1] S.-K. Ma, Modern theory of critical phenomena, Frontiers in Physics (Reading, MA: W. A. Benjamin, 1976).

[2] S. Sachdev, Quantum Phase Transitions (Cambridge University Press, 1999).

[3] U. C. Täuber, Critical Dynamics: A Field Theory Approach to Equilibrium and Non-Equilibrium Scaling Behavior (Cambridge University Press, 2014).

[4] M. Henkel, H. Hinrichsen, and S. Lübeck, NonEquilibrium Phase Transitions, Theoretical and Mathematical Physics, Vol. 1 (Springer, 2009).

[5] G.S. Agarwal, "Open quantum markovian systems and the microreversibility," Z. Phys. 258, 409-422 (1973).

[6] R. Chetrite and K. Mallick, "Quantum fluctuation relations for the lindblad master equation," J. Stat. Phys. 148, 480-501 (2012).

[7] L. M. Sieberer, A. Chiocchetta, A. Gambassi, U. C. Täuber, and S. Diehl, "Thermodynamic equilibrium as a symmetry of the schwinger-keldysh action," Phys. Rev. B 92, 134307 (2015)

[8] L. M. Sieberer, S. D. Huber, E. Altman, and S. Diehl, "Nonequilibrium functional renormalization for drivendissipative bose-einstein condensation," Phys. Rev. B 89, 134310 (2014).

[9] P. C. Hohenberg and B. I. Halperin, "Theory of dynamic critical phenomena," Rev. Mod. Phys. 49, 435 (1977).

[10] H. K. Janssen, B. Schaub, and B. Schmittmann, "New universal short-time scaling behaviour of critical relaxation processes," Z. Phys. B 73, 539 (1989).

[11] Haye Hinrichsen, "Non-equilibrium critical phenomena and phase transitions into absorbing states," Adv. Phys. 49, 815 (2000).

[12] Géza Ódor, "Universality classes in nonequilibrium lattice systems," Rev. Mod. Phys. 76, 663-724 (2004).

[13] Z. Racz, "Nonequilibrium Phase Transitions," eprint
arXiv:cond-mat/0210435 (2002), cond-mat/0210435

[14] Mehran Kardar, Giorgio Parisi, and Yi-Cheng Zhang, "Dynamic scaling of growing interfaces," Phys. Rev. Lett. 56, 889-892 (1986).

[15] Haye Hinrichsen, "On possible experimental realizations of directed percolation," Brazilian Journal of Physics 30, $69-82$ (2000).

[16] Kazumasa A. Takeuchi, Masafumi Kuroda, Hugues Chaté, and Masaki Sano, "Directed percolation criticality in turbulent liquid crystals," Phys. Rev. Lett. 99, 234503 (2007).

[17] Kazumasa A. Takeuchi, Masafumi Kuroda, Hugues Chaté, and Masaki Sano, "Experimental realization of directed percolation criticality in turbulent liquid crystals," Phys. Rev. E 80, 051116 (2009).

[18] M Marcuzzi, E Levi, W Li, J P Garrahan, B Olmos, and I Lesanovsky, "Non-equilibrium universality in the dynamics of dissipative cold atomic gases," New Journal of Physics 17, 072003 (2015).

[19] Marco Mattioli, Alexander W Glätzle, and Wolfgang Lechner, "From classical to quantum non-equilibrium dynamics of rydberg excitations in optical lattices," New Journal of Physics 17, 113039 (2015)

[20] Iacopo Carusotto and Cristiano Ciuti, "Quantum fluids of light," Rev. Mod. Phys. 85, 299-366 (2013).

[21] M. J. Hartmann, F. G. S. L. Brandão, and M. B. Plenio, "Quantum many-body phenomena in coupled cavity arrays," Laser Photonics Rev. 2, 527-556 (2008)

[22] A. Tomadin and Rosario Fazio, "Many-body phenomena in QED-cavity arrays," J. Opt. Soc. Am. B 27, A130A136 (2010)

[23] P. Schauß, J. Zeiher, T. Fukuhara, S. Hild, M. Cheneau, T. Macrì, T. Pohl, I. Bloch, and C. Gross, "Crystallization in ising quantum magnets," Science 347, 1455-1458 (2015), http://www.sciencemag.org/content/347/6229/1455.full.pdf.

[24] Helmut Ritsch, Peter Domokos, Ferdinand Brennecke, and Tilman Esslinger, "Cold atoms in cavity-generated dynamical optical potentials," Rev. Mod. Phys. 85, 553601 (2013)

[25] F. Brennecke, R. Mottl, K. Baumann, R. Landig, T. Donner, and T. Esslinger, "Real-time observation of fluctuations at the drivendissipative dicke phase transition," Proc. Natl. Acad. Sci. 110, 11763-11767 (2013)

[26] F. Nogrette, H. Labuhn, S. Ravets, D. Barredo, L. Béguin, A. Vernier, T. Lahaye, and A. Browaeys, "Single-atom trapping in holographic $2 \mathrm{~d}$ arrays of microtraps with arbitrary geometries," Phys. Rev. X 4, 021034 (2014)

[27] Malte Schlosser, Sascha Tichelmann, Jens Kruse, and Gerhard Birkl, "Scalable architecture for quantum information processing with atoms in optical microstructures," Quantum Information Processing 10, 907924 (2011)

[28] R. Dumke, M. Volk, T. Müther, F. B. J. Buchkremer, G. Birkl, and W. Ertmer, "Micro-optical realization of arrays of selectively addressable dipole traps: A scalable configuration for quantum computation with atomic qubits," Phys. Rev. Lett. 89, 097903 (2002).

[29] T.F. Gallagher, Rydberg Atoms (Cambridge University Press, 1984).

[30] M. Saffman, T. G. Walker, and K. Mølmer, "Quantum information with rydberg atoms," Rev. Mod. Phys. 82, 2313-2363 (2010). 
[31] R. Löw, H. Weimer, J. Nipper, J. B. Balewski, B. Butscher, H. P. Büchler, and T. Pfau, "An experimental and theoretical guide to strongly interacting Rydberg gases," J. Phys. B: At. Mol. Opt. Phys. 45, 113001 (2012).

[32] C. Carr, R. Ritter, C. G. Wade, C. S. Adams, and K. J. Weatherill, "Nonequilibrium phase transition in a dilute rydberg ensemble," Phys. Rev. Lett. 111, 113901 (2013)

[33] M. M. Valado, C. Simonelli, M. D. Hoogerland, I. Lesanovsky, J. P. Garrahan, E. Arimondo, D. Ciampini, and O. Morsch, "Experimental observation of controllable kinetic constraints in a cold atomic gas," Phys. Rev. A 93, 040701 (2016)

[34] A. Urvoy, F. Ripka, I. Lesanovsky, D. Booth, J. P. Shaffer, T. Pfau, and R. Löw, "Strongly correlated growth of rydberg aggregates in a vapor cell," Phys. Rev. Lett. 114, $203002(2015)$

[35] M. D. Lukin, M. Fleischhauer, R. Cote, L. M. Duan, D. Jaksch, J. I. Cirac, and P. Zoller, "Dipole blockade and quantum information processing in mesoscopic atomic ensembles," Phys. Rev. Lett. 87, 037901 (2001).

[36] Michael Hoening, Wildan Abdussalam, Michael Fleischhauer, and Thomas Pohl, "Antiferromagnetic longrange order in dissipative rydberg lattices," Phys. Rev. A 90, 021603 (2014)

[37] Lukas M. Sieberer, Michael Buchhold, and Sebastian Diehl, "Keldysh field theory for driven open quantum systems," arXiv:1512.00637 (2015).

[38] Aditi Mitra, So Takei, Yong Baek Kim, and A. J. Millis, "Nonequilibrium quantum criticality in open electronic systems," Phys. Rev. Lett. 97, 236808 (2006)

[39] E. G. Dalla Torre, E. Demler, T. Giamarchi, and E. Altman, "Quantum critical states and phase transitions in the presence of non-equilibrium noise," Nature Physics 6, 806 (2010).

[40] Emanuele G. Dalla Torre, Sebastian Diehl, Mikhail D. Lukin, Subir Sachdev, and Philipp Strack, "Keldysh approach for nonequilibrium phase transitions in quantum optics: Beyond the dicke model in optical cavities," Phys. Rev. A 87, 023831 (2013).

[41] L. M. Sieberer, S. D. Huber, E. Altman, and S. Diehl, "Dynamical critical phenomena in driven-dissipative systems," Phys. Rev. Lett. 110, 195301 (2013).

[42] A. Mitra and T. Giamarchi, "Mode coupling induced dissipative and thermal effects at long times after a quantum quench," Phys. Rev. Lett. 107, 150602 (2011).

[43] A. Mitra and T. Giamarchi, "Thermalization and dissipation in out of equilibrium quantum systems: A perturbative renormalization group approach," Phys. Rev. B 85, 075117 (2012).

[44] Ehud Altman, Lukas M. Sieberer, Leiming Chen, Sebastian Diehl, and John Toner, "Two-dimensional superfluidity of exciton polaritons requires strong anisotropy," Phys. Rev. X 5, 011017 (2015).

[45] T. Prosen and Marko Znidarič, "Long-range order in nonequilibrium interacting quantum spin chains," Phys. Rev. Lett. 105, 060603 (2010)

[46] Tony E. Lee, Sarang Gopalakrishnan, and Mikhail D. Lukin, "Unconventional magnetism via optical pumping of interacting spin systems," Phys. Rev. Lett. 110, 257204 (2013)

[47] Jamir Marino and Sebastian Diehl, "Driven markovian quantum criticality," Phys. Rev. Lett. 116, 070407 (2016).
[48] G. Lindblad, "On the generators of quantum dynamical semigroups," Communications in Mathematical Physics 48, 119-130 (1976)

[49] H.P. Breuer and F. Petruccione, The Theory of Open Quantum Systems (OUP Oxford, 2007).

[50] Merlijn van Horssen, Emanuele Levi, and Juan P. Garrahan, "Dynamics of many-body localization in a translation-invariant quantum glass model," Phys. Rev. B 92, 100305 (2015)

[51] J. M. Hickey, S. Genway, and J. P. Garrahan, "Signatures of many-body localisation in a system without disorder and the relation to a glass transition," ArXiv e-prints (2014), arXiv:1405.5780 [cond-mat.stat-mech].

[52] M.O. Scully and M.S. Zubairy, Quantum Optics (Cambridge University Press, 1997).

[53] See Supplemental Material, which includes Refs. [54,55].

[54] M. F. Maghrebi and A. V. Gorshkov, Phys. Rev. B 93, 014307 (2016)

[55] A. Altland and B. D. Simons, Condensed Matter Field Theory, 2nd ed. (Cambridge University Press, 2010).

[56] P. C. Martin, E. D. Siggia, and H. A. Rose, "Statistical dynamics of classical systems," Phys. Rev. A 8, 423 (1973).

[57] H. K. Janssen, "On a lagrangean for classical field dynamics and renormalization group calculations of dynamical critical properties," Z. Phys. B 23, 377 (1976).

[58] C. de Dominicis, "Techniques de renormalisation de la théorie des champs et dynamique des phénomènes critique," J. Phys. Colloq. (Paris) 37, 247 (1976)

[59] A. Kamenev, Field Theory of Non-Equilibrium Systems (Cambridge University Press, 2011).

[60] H. D. Abarbanel, J. D. Bronzan, R. L. Sugar, and A. R. White, Phys. Rep. 21, 119 (1975).

[61] M. B. Plenio and P. L. Knight, "The quantum-jump approach to dissipative dynamics in quantum optics," Rev. Mod. Phys. 70, 101-144 (1998)

[62] M. Viteau, M. G. Bason, J. Radogostowicz, N. Malossi, D. Ciampini, O. Morsch, and E. Arimondo, "Rydberg excitations in bose-einstein condensates in quasione-dimensional potentials and optical lattices," Phys. Rev. Lett. 107, 060402 (2011).

[63] H. Schempp, G. Günter, M. Robert-de-Saint-Vincent, C. S. Hofmann, D. Breyel, A. Komnik, D. W. Schönleber, M. Gärttner, J. Evers, S. Whitlock, and M. Weidemüller, "Full counting statistics of laser excited Rydberg aggregates in a one-dimensional geometry," Phys. Rev. Lett. 112, 013002 (2014)

[64] N. Malossi, M. M. Valado, S. Scotto, P. Huillery, P. Pillet, D. Ciampini, E. Arimondo, and O. Morsch, "Full counting statistics and phase diagram of a dissipative rydberg gas," Phys. Rev. Lett. 113, 023006 (2014)

[65] V. Gavryusev, A. Signoles, M. Ferreira-Cao, G. Zürn, C. S. Hofmann, G. Günter, H. Schempp, M. Robert-deSaint-Vincent, S. Whitlock, and M. Weidemüller, "Density matrix reconstruction of three-level atoms via Rydberg electromagnetically induced transparency," ArXiv e-prints (2016), arXiv:1602.07728 [physics.atom-ph].

[66] T. Baluktsian, B. Huber, R. Löw, and T. Pfau, "Evidence for strong van der waals type rydberg-rydberg interaction in a thermal vapor," Phys. Rev. Lett. 110, 123001 (2013).

[67] T. Wilk, A. Gaëtan, C. Evellin, J. Wolters, Y. Miroshnychenko, P. Grangier, and A. Browaeys, "Entanglement of two individual neutral atoms using rydberg blockade," 
Phys. Rev. Lett. 104, 010502 (2010).

[68] D. Barredo, H. Labuhn, S. Ravets, T. Lahaye, A. Browaeys, and C. S. Adams, "Coherent excitation transfer in a spin chain of three rydberg atoms," Phys. Rev. Lett. 114, 113002 (2015)

[69] Henning Labuhn, Sylvain Ravets, Daniel Barredo, Lucas Béguin, Florence Nogrette, Thierry Lahaye, and Antoine Browaeys, "Single-atom addressing in microtraps for quantum-state engineering using rydberg atoms," Phys. Rev. A 90, 023415 (2014).

[70] H. Labuhn, D. Barredo, S. Ravets, S. de Léséleuc, T. Macrì, T. Lahaye, and A. Browaeys, "A highlytunable quantum simulator of spin systems using twodimensional arrays of single Rydberg atoms," ArXiv eprints (2015), arXiv:1509.04543 [cond-mat.quant-gas],

[71] Igor Lesanovsky and Juan P. Garrahan, "Out-ofequilibrium structures in strongly interacting rydberg gases with dissipation," Phys. Rev. A 90, 011603 (2014).

[72] Beatriz Olmos, Igor Lesanovsky, and Juan P. Garrahan, "Out-of-equilibrium evolution of kinetically constrained many-body quantum systems under purely dissipative dynamics," Phys. Rev. E 90, 042147 (2014).

[73] Igor Lesanovsky and Juan P. Garrahan, "Kinetic con- straints, hierarchical relaxation, and onset of glassiness in strongly interacting and dissipative rydberg gases," Phys. Rev. Lett. 111, 215305 (2013).

[74] C. Ates, T. Pohl, T. Pattard, and J. M. Rost, "Antiblockade in rydberg excitation of an ultracold lattice gas," Phys. Rev. Lett. 98, 023002 (2007).

[75] Thomas Amthor, Christian Giese, Christoph S. Hofmann, and Matthias Weidemüller, "Evidence of antiblockade in an ultracold rydberg gas," Phys. Rev. Lett. 104, 013001 (2010)

[76] D. F. Walls and G. J. Milburn, "Effect of dissipation on quantum coherence," Phys. Rev. A 31, 2403-2408 (1985).

[77] Peter Degenfeld-Schonburg and Michael J. Hartmann, "Self-consistent projection operator theory for quantum many-body systems," Phys. Rev. B 89, 245108 (2014).

[78] M Marcuzzi, J Schick, B Olmos, and I Lesanovsky, "Effective dynamics of strongly dissipative rydberg gases," J. Phys. A: Math. Theor. 47, 482001 (2014).

[79] Alessio Chiocchetta, Marco Tavora, Andrea Gambassi, and Aditi Mitra, "Short-time universal scaling in an isolated quantum system after a quench," Phys. Rev. B 91, $220302(2015)$ 